\section{Envenenamentos acidentais entre menores de 15 anos em município da Região Sul do Brasil}

\author{
Accidental poisoning among children \\ and adolescents in a county in southern Brazil
}

\section{Abstract}

1 Centro de Ciências da Saúde, Universidade Estadual de Londrina, Londrina, Brasil.

Correspondência C. B. G. Martins Centro de Ciências da Saúde, Departamento de Enfermagem, Universidade Estadual de Londrina. Rua Chile 185, apto. 103 Londrina, $P R$ 86010-220, Brasil. leocris2001@aol.com
This study analyzes the characteristics of accidental poisoning among children and young adolescents (< 15 years) in Londrina, Paraná State, Brazil, in 2001. Data were obtained from patient records in general hospitals, the local Poison Control Center, and the municipal Mortality Information Center. There were 473 cases of poisoning, with an incidence rate of 339.8 per 100,000, 58.5\% due to poisonous substances and $39 \%$ due to venomous animals and plants. Increased risk was observed in the 1-3-year age group, due to poisonous substances. Medications (47.5\%), pesticides (14.1\%), and cleaning products (11.3\%) were the main substances involved (284 cases), and $17.2 \%$ of children were hospitalized. Among 189 cases of contact with venomous animals and plants, caterpillars (29.1\%), bees (25.9\%), and spiders (22.8\%) were the main agents, with a $1.1 \%$ hospitalization rate. Incidence of poisoning is high, and there are important differences in the types of agents involved according to age group, which may help orient the prevention of such accidents.

Morbidity; Toxic Substances; Poisoning
Christine Baccarat de Godoy Martins 1

Selma Maffei de Andrade 1

Priscila Aparecida Batista de Paiva 1

\section{Introdução}

Os acidentes domésticos entre crianças são freqüentes e contribuem para elevar a morbimortalidade infantil. Os envenenamentos (ou intoxicações) representam um dos principais tipos de acidente envolvendo crianças e alcançam uma dimensão preocupante. Na sua grande maioria, são considerados acidentais, porém decorrem de situações facilitadoras, de características peculiares às fases da criança, de comportamentos inadequados da família e do pouco incentivo às medidas preventivas 1 .

Nos últimos anos, tem crescido a diversidade de produtos farmacológicos e sanitários com alto poder tóxico, alguns com embalagens inadequadas e sem informações necessárias sobre composição, medidas preventivas e de tratamento em caso de acidente. Muitos, além disso, chamam a atenção das crianças pelo conteúdo colorido e estético, favorecendo a ocorrência de intoxicações 2 .

Segundo o Sistema Nacional de Informações Tóxico-Farmacológicas (SINITOX) 3, em 2002, 25 dos 33 Centros de Informação e Assistência Toxicológica em atividade no Brasil registraram 75.212 casos de intoxicação humana, com maiores letalidades nos casos de intoxicação por agrotóxicos de uso agrícola $(2,3 \%)$ e raticidas $(1,4 \%)$. A faixa etária mais acometida foi a de menores de cinco anos $(25,4 \%)$. Com relação aos principais agentes causadores de into- 
xicação entre os menores de cinco anos, destacam-se os medicamentos $(37,4 \%)$, os produtos domissanitários $(18,8 \%)$ e os produtos químicos industriais $(8,4 \%)$. No Brasil, os óbitos registrados por envenenamento (375 mortes em 2002) correspondem a $66,9 \%$ do total de óbitos registrados no país, segundo o SINITOX.

Entre os envenenamentos na infância, destacam-se também os causados por animais peçonhentos e plantas venenosas. No Brasil, foram registrados, em 2002, 17.977 envenenamentos por animais peçonhentos, dentre os quais 6.473 (36\%) decorrentes de contato com escorpiões, 5.294 (29,4\%), com serpentes, e 3.538 $(19,7 \%)$, com aranhas 3 . Estudo de Bucaretchi et al. de 20024 mostra que em um hospital-escola de Campinas, São Paulo, Brasil, num prazo de 13 anos, 31 menores de 15 anos de idade foram admitidos vítimas de picada da serpente Crotalus durissus ssp., mais da metade dos quais apresentou manifestações clínicas graves $(58,1 \%)$. Outro estudo 5 revela que o período de maior ocorrência de acidentes com serpentes entre menores de 15 anos, na região de Campinas, vai de outubro a março (período de maiores temperatura e umidade), atinge predominantemente os membros inferiores e tem a Bothrops jararaca como espécie mais identificada.

Diante desse contexto, torna-se fundamental a realização de estudos que possam caracterizar os envenenamentos acidentais e suas vítimas com vistas a melhor direcionar os esforços para o seu controle. Portanto, a presente investigação teve como objetivo analisar as características epidemiológicas dos envenenamentos acidentais entre menores de 15 anos no Município de Londrina, Paraná, Brasil.

\section{Metodologia}

A pesquisa se constituiu em um estudo transversal e descritivo acerca da morbi-mortalidade por envenenamento (intoxicação) acidental entre menores de 15 anos. A população de estudo foi composta por menores de 15 anos de idade residentes no Município de Londrina, vítimas de envenenamento no período de 1 o de janeiro a 31 de dezembro de 2001 e que receberam assistência hospitalar em nível de pronto-socorro ou internação, assistência do Centro de Controle de Intoxicações (CCI) do município ou que morreram em conseqüência de envenenamento acidental.

Para identificação dos casos, foram levantados manualmente todos os prontuários de menores de 15 anos, bem como as fichas de atendimento prestado a eles em pronto-socor- ro ou em internação nos cinco hospitais gerais da cidade que atendem a essa faixa etária, em 2001. Em seguida, foram separados e transcritos, para formulário específico, todos os casos referentes a causas externas (acidentes e violências) de menores de 15 anos residentes na cidade, atendidos ou não pelo Sistema Único de Saúde (SUS). Dentre esses atendimentos por causa externa, foram selecionados, para o presente trabalho, os casos com referência a intoxicação (envenenamento) acidental. Da mesma forma, os registros constantes no CCI, localizado dentro de um hospital de ensino, foram consultados, com separação e transcrição dos casos de atendimento de menores de 15 anos, residentes no município, identificando, também para este estudo, os casos de intoxicação (envenenamento) acidental. Dentre essas instituições, apenas o CCI possui fichas padronizadas para o atendimento de intoxicações; nas demais, buscou-se o termo "intoxicação" ou "envenenamento" nos registros de atendimento médico nas fichas de pronto-socorro ou de internação. Para a coleta de dados nos prontuários selecionados, foi utilizado um formulário com 21 questões fechadas, composto de quatro partes: dados de identificação da criança, dados referentes ao acidente, dados referentes ao atendimento e conseqüências do acidente. O instrumento foi previamente testado com dados de atendimento do ano de 2000 e utilizado na coleta de dados nos próprios hospitais por uma equipe de alunos do curso de enfermagem da Universidade Estadual de Londrina, previamente treinados e supervisionados por uma das autoras do estudo.

Além dos dados de morbidade, foram também analisadas as declarações de óbito de menores de 15 anos nos anos de 2001 e 2002, a fim de verificar óbitos em conseqüência de envenenamento que pudessem ter ocorrido em $2001 \mathrm{e}$ que não receberam assistência médica ou de óbitos que tivessem acontecido até um ano após o acidente ocorrido em 2001, conforme recomenda a Classificação Estatística Internacional de Doenças e Problemas Relacionados à Saúde, 10a revisão ${ }^{6}$. Os dados de mortalidade foram coletados no Núcleo de Informação em Mortalidade da Prefeitura de Londrina (NIM), o qual investiga rotineiramente todos os casos de óbito por causa externa do tipo ignorado.

O tipo de intoxicação foi classificado conforme o capítulo XX da CID-10 6. Nessa classificação, há alguns agrupamentos de causas relacionados a intoxicações (envenenamentos). Entre os eventos acidentais, são descritos os envenenamentos por exposição a substância nociva (códigos X40 a X49) e os contatos com 
animais ou plantas venenosos (X20 a X29). Entre os eventos intencionais são apresentadas as lesões autoprovocadas por medicamentos ou outros produtos (códigos X60 a X69) e as agressões (códigos X85 a X90). Há ainda um agrupamento de códigos residuais, em que são classificados os envenenamentos em que se ignora se foram acidentais ou intencionais (códigos Y10 a Y19). Para o presente estudo, foram selecionadas apenas as causas acidentais, por exposição a substância tóxica ou por contato com animais ou plantas venenosas.

Todos os dados obtidos foram processados eletronicamente e tabulados por meio do programa computacional Epi Info versão 6.04d (Centers for Disease Control and Prevention, Atlanta, Estados Unidos). Foi realizada exaustiva verificação de inconsistências mediante cruzamento e verificação de dados. Registros duplicados referentes a um mesmo acidente, por exemplo, no caso de uma criança atendida em dois hospitais, foram considerados como um único caso, a fim de se calcular o número de acidentes e não o de atendimentos.

Para fins de comparação dos dados obtidos com os dados dos sistemas oficiais de informação, foram também levantados, para os menores de 15 anos residentes em Londrina: (a) os casos detectados pelo CCI; (b) as internações registradas no Sistema de Informação Hospitalar do Sistema Único de Saúde (SIH-SUS), cujos diagnósticos principais estivessem codificados como intoxicação (códigos T36 a T65 da CID-10) ou cujos diagnósticos secundários mencionassem envenenamento acidental (códigos de X20 a X29 ou códigos de X40 a X49 da CID-10), e (c) os óbitos por envenenamento acidental (códigos de X20 a X29 ou de X40 a X49 da CID-10) registrados no Sistema de Informação em Mortalidade (SIM).

Solicitou-se aos diretores ou responsáveis de cada instituição a autorização para acesso aos prontuários e informações. O projeto de pesquisa foi apreciado e aprovado pelo Comitê de Ética em Pesquisa da Universidade Estadual de Londrina.

\section{Resultados}

O total de casos de envenenamento acidental na população de estudo foi de 473 , o que correspondeu a um coeficiente de incidência de 399,8 por 100 mil crianças dessa faixa etária e a $5,3 \%$ dos 8.854 atendimentos hospitalares (pronto-socorro e internações) e/ou óbitos por causas externas na mesma população e faixa etária, em 2001.
Com relação aos tipos de envenenamento, classificados de acordo com o capítulo XX da CID-10, 60\% ocorreram por exposição a substância nociva (códigos X40 a X49), com uma incidência de 240,1 por 100 mil crianças, e $40 \%$ por contato com animais e plantas venenosos (códigos X20 a X29), com incidência de 159,8 por $100 \mathrm{mil}$.

A Figura 1 mostra os coeficientes de incidência por faixa etária, em relação a esses dois tipos de envenenamento. A faixa etária de maior risco para acidentes por exposição a substâncias nocivas foi a de um a três anos. Não houve grande variação, após um ano de idade, quanto à incidência de acidentes por contato com planta ou animal venenosos.

Os acidentes por envenenamento devido a exposição a substância nociva foram observados com maior freqüência no sexo feminino $(52,1 \%)$, ao passo que o sexo masculino predominou entre os casos de acidentes por contato com planta ou animal venenosos $(68,3 \%)$.

Dos 473 menores de 15 anos vítimas de envenenamento, $422(89,2 \%)$ foram atendidos exclusivamente em pronto-socorro e 51 foram internados, resultando numa taxa de internação igual a $10,8 \%$. Não foram, porém, verificados óbitos por intoxicação, mesmo observando-se um prazo de até um ano após.

Também não houve óbitos detectados pelo SIM. Comparando os casos constatados no estudo com os do CCI, observou-se que houve registro, neste centro, de 275 casos (180 por exposição a substância nociva e 95 por contato com animal ou planta venenosos), o que representa $58,1 \%$ do total de casos identificados no estudo, $63,3 \%$ dos envenenamentos por substância nociva e 50,3\% dos por contato com animal ou planta venenosos. Relativamente aos casos internados, enquanto o presente trabalho identificou 51 casos, o CCI registrou 36 (70,6\% do observado no estudo) e o SIH-SUS apenas 11 (21,6\% dos 51 internados e $23,9 \%$ dos 46 internados pelo SUS, identificados no estudo).

Dos 284 casos de acidentes por exposição a substância nociva identificados neste estudo, quase a metade das intoxicações se deveu a medicamentos. Quatro tipos de agentes (medicamentos, pesticidas, produtos de limpeza e plantas/frutos) corresponderam a $82,7 \%$ dos casos (Tabela 1).

Quanto ao nível de atendimento, 235 (82,7\%) crianças vítimas de acidentes por exposição a substância nociva foram atendidas somente em pronto-socorro e 49 (17,2\%) necessitaram de internação. Para cada internação, aproximadamente cinco crianças foram atendidas em pronto-socorro. 
Figura 1

Coeficientes de incidência de envenenamento acidental em menores de 15 anos, segundo tipo de envenenamento

e faixa etária. Londrina, Paraná, Brasil, 2001.

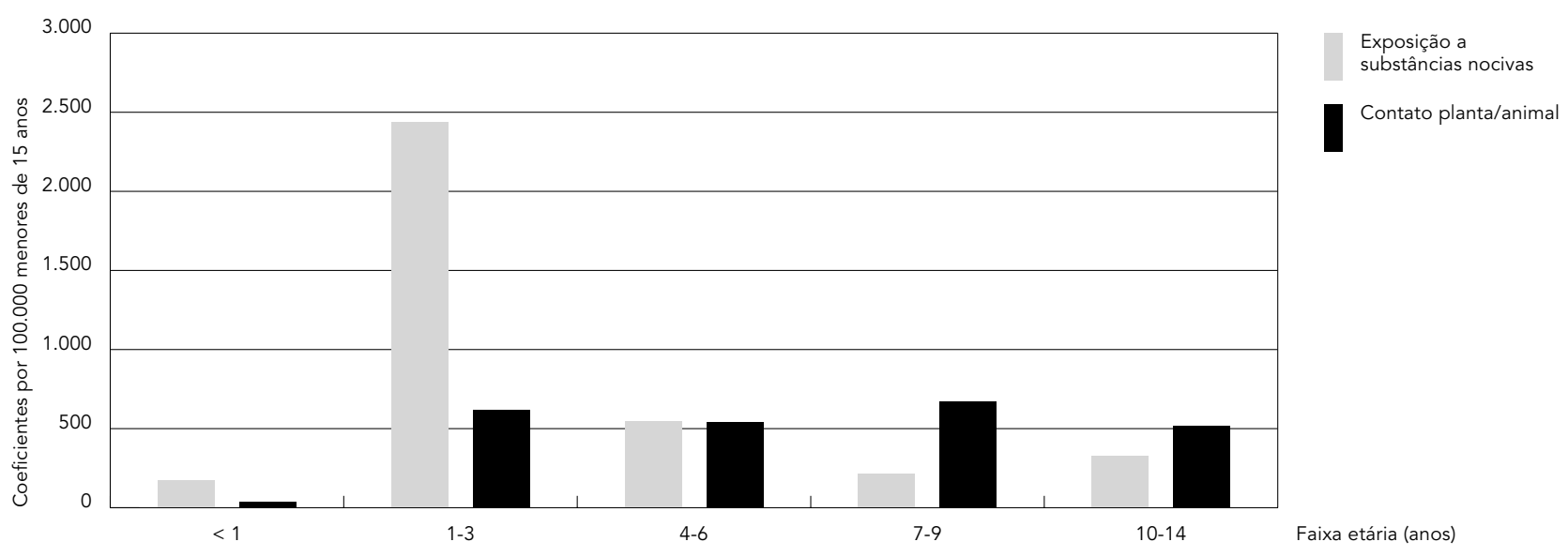

* Coeficientes por 100 mil menores de 15 anos.

Com relação aos acidentes por contato com animal ou planta venenosos, as lagartas foram as principais responsáveis, seguidas por abelhas e aranhas (Tabela 2). Para este tipo de acidente, não foi possível especificar o agente em $10,6 \%$ dos casos.

No que se refere ao nível de atendimento e evolução das vítimas de acidentes por contato com animal ou planta venenosos, 187 (98,9\%) crianças foram atendidas em pronto-socorro e $2(1,1 \%)$ foram internadas. Para cada internação, aproximadamente 94 crianças foram atendidas no pronto-socorro.

\section{Discussão}

Os acidentes por envenenamento desempenham um papel importante no contexto dos acidentes na infância, por sua alta freqüência, custos de tratamento, possibilidade de seqüelas irreversíveis e pelo sofrimento que causam às crianças e suas famílias 7 . Estudos detalhados podem contribuir para a adoção de ações que revertam o quadro de alta incidência observado, por meio de medidas específicas a cada fase do desenvolvimento infantil.

Esta investigação pretendeu trazer alguns conhecimentos adicionais sobre a morbidade por envenenamentos acidentais entre menores de 15 anos, temática ainda pouco explorada em publicações nacionais, apesar da impor- tância epidemiológica desse agravo. Tem como ponto a se destacar, em relação a outros estudos, o fato de que a análise dos dados não se ateve apenas aos casos notificados ou registrados nos sistemas oficiais de informação, pois houve, também, busca ativa em todos os registros de pronto-socorro, internação e óbitos de crianças menores de 15 anos residentes na cidade, atendidas ou não pelo setor público. Este método tende a aproximar do real o coeficiente de incidência encontrado, embora, ainda, certamente subestimado, em virtude da grande parcela da população que não busca serviços de saúde hospitalares para a resolução de seus problemas de saúde, além da falta de uniformidade nos registros médico-hospitalares, que pode levar a sub-registro.

Por outro lado, os resultados do estudo revelam a necessidade de se estabelecerem estratégias que visem à melhoria da qualidade e cobertura dos registros sobre morbidade por envenenamentos rotineiramente coletados, como os que compõem a informação do SIH-SUS e do CCI, para que estas informações possam ser usadas, de forma mais efetiva, no monitoramento das ocorrências desses acidentes, tornando dispensáveis, assim, levantamentos trabalhosos, como o realizado nesta investigação.

Não foram encontrados, na revisão da literatura nacional, estudos que pudessem servir para comparação dos valores dos coeficientes de incidência, mesmo referente apenas à par- 
cela que recebe atenção hospitalar (pronto-socorro ou internação). Observou-se, ainda assim, no presente trabalho, que é alta a incidência de envenenamentos entre menores de 15 anos. A título de comparação, em Antígua e Barbuda, no Caribe, em levantamento semelhante ao do presente estudo, a incidência de envenenamentos por exposição a substâncias nocivas entre crianças menores de 13 anos foi de 1,3 por mil crianças (ou 130 por $100 \mathrm{mil}$ ) 8 , praticamente metade da observada na presente investigação (240,1 por 100 mil).

No presente estudo, observou-se maior risco desses acidentes entre crianças de um a três anos de idade, o que, possivelmente, deve-se ao seu grau de desenvolvimento. Nessa fase, as crianças são atraídas por produtos coloridos, caixas com comprimidos que parecem balas e líquidos coloridos, levando-os, freqüentemente, à boca. Aliado a esses atrativos, tem-se o fato de que a criança, nessa fase, começa a subir em móveis, alcançando prateleiras e gavetas com medicamentos e produtos e, também, abrindo os armários, numa atividade de intensa exploração do meio. O contato com substâncias tóxicas ocorre, assim, com facilidade, principalmente no ambiente da cozinha, banheiro, lavanderia e quintal 2,7,8,9,10.

Resultados similares aos do presente estudo foram relatados por outros trabalhos. Baracat el al. 9 observaram a intoxicação (ou envenenamento) como causa freqüente de acidentes entre crianças menores de 15 anos atendidas em um pronto-socorro de Campinas, São Paulo, Brasil, com $49,5 \%$ dos casos na faixa dos dois a cinco anos. Na região do Caribe 8 , as crianças de um a três anos foram as mais freqüentes, entre menores de 13 anos, atendidas por envenenamento no único hospital da região. No Brasil, cerca de um terço das intoxicações medicamentosas notificadas à Rede Nacional de Centros de Controle de Intoxicações se refere a crianças menores de cinco anos 10 .

Com relação ao agente causador da intoxicação, no Brasil, tal qual no presente estudo, os medicamentos têm sido identificados como os grandes responsáveis pelos acidentes entre crianças 9,10 . Bortoletto \& Bochner 10 constataram, entre casos notificados ao CCI no Brasil, os medicamentos $(26,5 \%)$, os pesticidas $(8,2 \%)$ e os produtos de limpeza $(7,3 \%)$ como os principais agentes responsáveis por envenenamento acidental, sendo os menores de cinco anos o principal grupo envolvido com intoxicação medicamentosa. Baracat et al. 9 também verificaram, entre menores de 15 anos, a intoxicação medicamentosa como o principal tipo de acidente deste grupo de causas, seguida pela in-

\begin{tabular}{|c|c|c|}
\hline \multicolumn{3}{|c|}{$\begin{array}{l}\text { Distribuição dos menores de } 15 \text { anos vítimas } \\
\text { de envenenamento acidental por exposição } \\
\text { a substância nociva, segundo o tipo de agente. } \\
\text { Londrina, Paraná, Brasil, } 2001 .\end{array}$} \\
\hline Agente causador & $\mathbf{n}$ & $\%$ \\
\hline Medicamento & 135 & 47,5 \\
\hline Pesticida & 40 & 14,1 \\
\hline Produto de limpeza & 32 & 11,3 \\
\hline Plantas ou frutos & 28 & 9,9 \\
\hline Soda cáustica & 14 & 4,9 \\
\hline Querosene & 8 & 2,8 \\
\hline Álcool & 5 & 1,8 \\
\hline Alimento & 5 & 1,8 \\
\hline Cosmético & 4 & 1,4 \\
\hline Outros & 5 & 1,8 \\
\hline Não especificado & 8 & 2,8 \\
\hline Total & 284 & $100,0^{*}$ \\
\hline
\end{tabular}

* Aproximado para 100,0\%.

Tabela 2

Distribuição dos menores de 15 anos vítimas de envenenamento acidental por contato com animal ou planta venenosos, segundo o tipo de agente. Londrina, Paraná, Brasil, 2001.

\begin{tabular}{lrr}
\hline Agente envolvido & $\mathbf{n}$ & $\%$ \\
\hline Lagarta & 55 & 29,1 \\
Abelha & 49 & 25,9 \\
Aranha & 43 & 22,8 \\
Planta & 11 & 5,8 \\
Inseto venenoso & 3 & 1,6 \\
Formiga & 3 & 1,6 \\
Escorpião & 3 & 1,6 \\
Cobra & 2 & 1,1 \\
Não especificado & 20 & 10,6 \\
Total & 189 & $100,0 *$ \\
\hline
\end{tabular}

* Aproximado para 100,0\%.

toxicação por produtos de limpeza. Bortoletto \& Bochner 10 destacam, ainda, que o medicamento é o principal agente que causa intoxicação em seres humanos em todo o país, ocupando o primeiro lugar nas estatísticas do SINITOX na década de 1990.

A prática de automedicação, a guarda de medicamentos ao alcance de crianças, o formato atraente, o gosto palatável e a embala- 
gem facilmente violável desses produtos podem ser fatores contribuintes para a elevada ocorrência de acidentes por intoxicação medicamentosa entre crianças $10,11,12$, as quais, dependendo da idade, possuem características de curiosidade e imitação de procedimentos realizados por adultos. Embora ainda em tramitação no Congresso Nacional, o projeto de lei que estabelece a "Embalagem Especial de Proteção à Criança - EEPC” poderia contribuir, sem dúvida, para a redução desses eventos, como observado em países desenvolvidos 10 . Outro fator a considerar nos casos de intoxicação por medicamento consiste na superdosagem ou troca de medicamentos, oferecidos à criança por engano. Orientações sistemáticas aos pais, fornecidas por serviços de saúde e farmácias, quanto à necessidade de verificação de rótulos, dosagens, manutenção dos remédios nas embalagens originais e administração de medicamentos em locais de boa visibilidade, poderiam ajudar a evitar erros e superdosagens.

Nos envenenamentos acidentais por domissanitários e pesticidas, uma questão a ser ponderada é a possibilidade de acesso fácil da criança a esses produtos, que, em geral, são guardados sob pias de cozinha ou em armários baixos, e a facilidade de abertura de seus recipientes pelas crianças 2 . Conquanto os acidentes devido à ingestão de soda cáustica tenham representado, no presente estudo, apenas $4,9 \%$ dos acidentes por exposição a substâncias nocivas, a possibilidade de seqüelas permanentes resultantes de queimadura de órgãos internos indica a necessidade de adoção de estratégias que evitem sua ocorrência, tais como a venda do produto mediante recomendações quanto aos riscos da ingestão, a implantação de lacres de segurança e a orientação quanto à guarda em local seguro.

Os envenenamentos acidentais por exposição a substâncias nocivas guardam, portanto, relação direta com o ambiente doméstico, com a fase de desenvolvimento da criança e com as características dos produtos industriais 10 . No dormitório, a criança pode encontrar remédios, perfumes, cosméticos, entre outros; no banheiro, a existência de produtos de higiene e de limpeza favorece a ocorrência de casos acidentais de envenenamento; na cozinha, a criança depara, com facilidade, com sabão, detergente, produtos de limpeza, bebidas alcoólicas e outros; na área de serviço, as ceras, solventes, inseticidas, raticidas, álcool, fertilizantes, alvejantes, entre outros produtos, constituem um verdadeiro arsenal para intoxicações acidentais. Deve-se considerar, também, o acondicionamento de produtos tóxicos, como gasolina e produtos diversos, em garrafas plásticas de refrigerante, o que pode favorecer a ocorrência de ingestão acidental por crianças. Outro fator a ser destacado é o contato das crianças com lixo acumulado a céu aberto, principalmente nas periferias da cidade, o que facilita o contato com substâncias tóxicas e produtos químicos. Dessa forma, é possível evitar o envenenamento acidental por meio de mudanças comportamentais e do ambiente, assim como por medidas legislativas que promovam a produção de medicamentos e produtos com consistência e embalagens mais seguras à violação por parte de crianças, especialmente das menores de cinco anos.

Os acidentes por contato com animal ou planta venenosos, embora incidam com menor freqüência entre crianças, apresentam, em geral, grande gravidade 13. No presente estudo, não houve grande variabilidade na incidência após um ano de idade, com valores superiores a 500 por 100 mil crianças.

O tipo de agente causador do acidente por animal peçonhento depende, entre outros fatores, do clima e das condições sociais de cada localidade. Na presente investigação, lagartas, abelhas e aranhas foram os principais tipos de agentes causadores do acidente. Em Londrina, predominam dias ensolarados, com altas temperaturas e baixos índices pluviométricos em grande parte do ano, o que favorece, por exemplo, a instalação de colméias e a dispersão de abelhas, com conseqüente aumento do risco de acidentes com humanos 14 . Sabe-se, também, que na Região Sul, especialmente no Paraná e Santa Catarina, predominam aracnídeos do gênero Loxosceles, popularmente conhecidos como "aranha-marrom" 15. Não foi possível para este estudo, com base nos registros hospitalares, entretanto, conhecer o gênero ou espécie predominante de cada tipo de inseto ou animal peçonhento, dadas as características e condições em que esses registros são feitos. Também não foi possível conhecer o tipo de agente em 10,6\% dos casos desses acidentes, possivelmente pela dificuldade em apreensão ou identificação do animal venenoso após o acidente, embora a má qualidade do registro no atendimento também não possa ser descartada.

Cuidados simples e de baixo custo podem ser empregados para evitar acidentes por contato com animal ou planta venenosos, tais como conhecer as características das plantas que se têm em casa, mantê-las distantes do alcance da criança, manter o gramado devidamente aparado, exigir das autoridades competentes que mantenham limpos locais públicos próxi- 
mos às residências, sacudir e examinar calçados antes de usar, eliminar entulhos que servem de habitação para animais venenosos, cobrir frestas entre paredes de madeira que podem servir de abrigo para animais peçonhentos, além de observar sempre o local onde a criança anda e brinca 15. Considerando que os acidentes com plantas ou animais venenosos ocasionam reações locais e sistêmicas, muitas vezes fatais se não tiverem a devida assistência 16, cuidados simples como os apontados acima podem contribuir para a preservação da saúde de nossas crianças. Todavia, ações relativas ao meio-ambiente, de responsabilidade do poder público, que previnam a proliferação de insetos e outros animais peçonhentos também não deveriam ser negligenciadas, sendo o controle social fundamental para que as ações necessárias sejam implementadas.

\section{Resumo}

O estudo teve como objetivo analisar as características de envenenamentos acidentais entre menores de 15 anos residentes em Londrina, Paraná, Brasil, em 2001. Os dados foram obtidos em prontuários dos hospitais gerais, do Centro de Controle de Intoxicações e no Núcleo de Informação em Mortalidade da cidade. Foram observadas 473 vítimas de envenenamento acidental, representando um coeficiente de incidência de 399, 8 por 100 mil crianças, sendo 60\% por exposição a substância nociva e $40 \%$ por contato com planta ou animais venenosos. Maior risco foi observado na faixa de um a três anos, por exposição a substâncias nocivas. Entre os acidentes causados por exposição a substâncias nocivas (284 casos), predominaram os por medicamentos $(47,5 \%)$, pesticidas $(14,1 \%)$ e produtos de limpeza (11,3\%), com internação de 17,2\% das crianças. Entre os envenenamentos por contato com animais/plantas (189 casos), predominaram os causados por lagartas (29,1\%), abelhas (25,9\%) e aranhas (22,8\%), com internação de 1,1\%. Conclui-se que é alta a incidência de envenenamentos e que há diferenciais nos agentes envolvidos de acordo com a faixa etária da criança, o que pode contribuir para evitar esses eventos.
Apesar da baixa taxa de internação observada para os casos de acidentes por contato com plantas ou animais venenosos observada no presente trabalho $(1,1 \%)$, o atendimento às crianças vítimas desse tipo de contato envolve, muitas vezes, a administração de soros e medicações específicas, gerando custos hospitalares consideráveis. Prevenir esses eventos permitirá reduzir a demanda aos serviços de saúde, além de todo o estresse que crianças e famílias vivenciam nesses casos.

Pelos resultados apresentados neste estudo, observou-se que, além da alta incidência, há diferenciais nos agentes envolvidos de acordo com a faixa etária da criança, o que pode contribuir com estratégias que visem a evitar esses eventos. Portanto, há que se ressaltar a importância do monitoramento dessas causas e de novos estudos que aprofundem o conhecimento do tema e que contribuam para a redução desses acidentes na infância.

\section{Colaboradores}

C. B. G. Martins e S. M. Andrade participaram na concepção do trabalho e em todas as demais etapas. P. A. B. Paiva colaborou na coleta e tabulação dos dados e na redação da versão preliminar do artigo. Todos os autores participaram na revisão critica do trabalho e na aprovação da versão final. 


\section{Referências}

1. Souza LJEX, Barroso MGT. Acidente doméstico em crianças: abordagem conceitual. Acta Paul Enfermagem 1999; 12:70-7.

2. Ferreira A, Borelli E, Casoni A, Santos FM, Oliveira MLF. Acidentes infantis domésticos por produtos domissanitários registrados em centro de assistência toxicológica da Região Sul. Espaço para a Saúde 2001; 3. http://www.ccs.uel.br/espacoparasaude/v3n1/indice1.htm (acessado em 02/ Abr/2003).

3. Sistema Nacional de Informações Tóxico-Farmacológicas. Casos registrados de intoxicação humana e envenenamento. http://www.cict.fiocruz. br/intoxicacoeshumanas/2002/umanalise2002.h tm (acessado em 02/Jul/2005).

4. Bucaretchi F, Herrera SR, Hyslop S, Baracat EC, Vieira RJ. Snakebites by Crotalus durissus ssp in children in Campinas, São Paulo, Brazil. Rev Inst Med Trop São Paulo 2002; 44: 133-8.

5. Bucaretchi F, Herrera SRF, Hyslop S, Bacarat ECE, Vieira RJ. Snakebites by Bothrops ssp in children in Campinas, São Paulo, Brazil. Rev Inst Med Trop São Paulo 2001; 43:329-33.

6. Organização Mundial de Saúde. Classificação estatística de doenças e problemas relacionados à saúde. 10ạ revisão. 8ạ Ed. São Paulo: Edusp; 2000.

7. Aleixo ECS, Itinose AM. A experiência dos familiares durante a ocorrência da intoxicação infantil no Município de Maringá (PR). Espaço para a Saúde 2001; 3. http: / /www.ccs.uel.br/espacopara saude/v3n1/indice1.htm (acessado em 02/Abr/ 2003).
8. Martin TC, Brinkman W. The spectrum of accidental childhood poisoning in the Caribbean. Rev Panam Salud Pública 2002; 12:313-6.

9. Baracat ECE, Paraschin K, Nogueira RJN, Reis MC, Fraga AMA, Sperotto G. Acidentes com crianças e sua evolução na região de Campinas, SP. J Pediatr (Rio de J) 2000; 76:368-74.

10. Bortoletto ME, Bochner R. Impacto dos medicamentos nas intoxicações humanas no Brasil. Cad Saúde Pública 1999; 15:859-69.

11. Simões MJS, Farache Filho A. Consumo de medicamentos em região do Estado de São Paulo (Brasil), 1985. Rev Saúde Pública 1988; 22:494-9.

12. Loyola Filho AI, Uchoa E, Guerra HL, Firmo JOA, Lima-Costa MF. Prevalência e fatores associados à automedicação: resultados do projeto Bambuí. Rev Saúde Pública, 2002; 36:55-62.

13. Oliveira JS, Campos JA, Costa DM. Acidentes por animais peçonhentos na infância. J. Pediatr (Rio de J) 1999; 75 Suppl 2:S251-8.

14. Mello MHSH, Silva EA, Natal D. Abelhas africanizadas em área metropolitana do Sul do Brasil: abrigos e influências climáticas. Rev Saúde Pública 2003 ; 37:237-41.

15. Fundação Nacional de Saúde, Ministério da Saúde. Manual de diagnóstico e tratamento de acidentes por animais peçonhentos. Brasília: Fundação Nacional de Saúde, Ministério da Saúde; 2001.

16. Schvartsman C, Schvartsman S. Intoxicações exógenas agudas. J Pediatr (Rio de J) 1999; 75 Suppl 2:S244-50.

Recebido em 22/Mar/2005

Versão final reapresentada em 18/Jul/2005

Aprovado em 02/Set/2005 